\title{
RISCO GRAVIDÍCO E RISCO PERINATAL: IMPLICAÇÕES METODOLÓGICAS, TEÓRICAS E TÉCNICAS, DAS PROPOSTAS DE MENSURAÇÃO*
}

\author{
HIGH-RISK PREGNANCY AND PERINATAL RISK: \\ MEASURING PROPOSALS AND THEIR METHODOLOGICAL \\ IMPLICATIONS OF THEORETICAL AND TECHNICAL NATURE
}

Augusta Thereza de Alvarenga ${ }^{1}$

ALVARENGA, A. T. Risco Gravidico e Risco Perinatal: Implicações Metodologicas, Teóricas e Técnicas, das Propostas de Mensuração. Rev. Bras. Cresc. Des. Hum., São Paulo, IV(2), 1994.

Resumo: O presente trabalho versa sobre as relações existentes entre os conceitos de risco gravídico e risco perinatal amplamente empregados quer no campo da Medicina, especialmente na área da Obstetrícia, quer da Saúde Materno-Infantil, entendida como área pertencente ao campo da Saúde Pública ou Coletiva. A autora procura retraçar a emergência do emprego do conceito de risco gravídico no contexto dos processos de recomposição da prática médica, especialmente em relação ao movimento denominado de Medicina Comunitária, ocorrido nos anos 60 nos Estados Unidos da América do Norte, caracterizando, a partir daí, a forma típica assumida pelas propostas de mensuração dos níveis de risco gravídico, conhecidas como "Sistemas de Escore" Aponta um conjunto de questões de natureza metodológica - teórica e técnica - presentes nessas propostas de mensuração que colocam, ao campo da Perinatologia, desafios não somente do ponto de vista do conhecimento gerado, mas também das propostas de intervenção que tal instrumental encerra. Pela importância do risco gravídico e do risco perinatal como objetos de preocupa,cão da área Materno-Infantil a autora conclui pela relevância de um investimento intelectual, sobretudo da área da Perinatologia - que representa uma interface entre Obstetrícia e Pediatria - em novos instrumentais metodológicos capazes de tratar tais objetos não de forma redutora, mas "holística" e multidisciplinar.

Palabra-chave: gestação de alto risco, risco perinatal, baixo peso ao nascer, prematuridade, retardo de crescimento intra-uterino, morbimortalidade perinatal, mensuração de risco gravídico.

\footnotetext{
* Versão revista e ampliada da palestra proferida em mesa redonda sobre “Aspectos Metodológicos - Investigações Prioritárias” no campo da Perinatologia no "I Seminário Franco-Brasileiro de Metodologias de pesquisa em Saúde Perinatal”. Realizado de 9 a 11 de março de 1994 com a promoção da Faculdade de Saúde Pública da USP e do INSERM (Institut National de la Santé et de la Recherche Médicale).

1 Socióloga, Sanitarista, Doutora em Saúde Pública. Prof. Dr. do Departamento de Saúde Materno-Infantil da Faculdade de Saúde Pública da Universidade de São Paulo.

End.: Av. Dr. Arnaldo 715 sala 218, São Paulo - SP, CEP 01246-904 Fax: (011) 853.0240
} 
Summary: The present paper is about lhe existing relations between the concepts of high risk pregnancy and perinatal risk, widely used both in lhe field of Medicine (specially in Obstetrics) and in the field of Maternal-Child Health, the latter seen as an arca within the Colective or Public Health field. The author tries to trace the emergence of the use of the high-risk pregnancy concept in the context of recomposition of medical practice, especially in relation to the Community Medicine Movement that occurred during the 60's in the USA. From then on, the typical form,adopted by the high-risk pregnancy levei measuring proposals - known as "score systems" - is characterized. The author also presents a set of methodological issues, both theoretical and technical present in these measuring proposals. These issues present challenges within the scope of Perinatology, not only from the point of view of the generated knowledge, but also from the point of view of the intervention proposals inherent to that practice. Due to the importance of gravidic and perinatal risks as points of concern in the Maternal-Child area, the author considers as relevant the investment in new methodological tools capable of dealing with these concerns in non-reducing, holistic an multidisciplinary ways.

Key Words: high-risk pregnancy, perinatal risk, low weight, prematurity, intrauterine growth retardation, perinatal morbimortality, measurement of high risk pregnancy. 


\section{CARACTERIZANDO AS RELAÇÕES ENTRE RISCO GRAVÍDICO E RISCO PERINATAL}

'Risco gravídico' trata-se de um termo empregado na área Materno-Infantil para nomear um conceito cujas características definidoras encontram-se diretarnente vinculadas ao estudo das questões de mortalidade e de morbidade perinatais. Mais especificamente tratado como "alto risco gravídico”, ganha expressão na literatura especializada das áreas Médica e da Saúde Pública por encerrar, no cerne de sua proposta, uma preocupação que medeia interesses não somente da assistência individual, mas sobretudo coletiva.

Embora emerja na literatura especializada de maneira típica, a partir dos anos 60, ou seja, no interior do movimento de recomposição da prática médica denominado “Medicina Comunitária” tem suas raízes já na década dos 30, inserido num sistema teórico da prática médica, onde, às concepções de gestação normal e patológica passam a atrelar-se as noções de padrões de crescimento intra-uterino normal e patológico, estreitamente ligadas às noções de prematuridade e de insuficiência ponderal tratadas, num primeiro momento, indistintamente, como uma questão de baixo peso ao nascer.

Assim colocado, o que se observa na literatura é o falo de que, metodologicamente falando, as causas da prematuridade e da insuficiência ponderal, ao lado das de mortalidade fetal e do concepto nos primeiros dias de vida, são buscadas, de maneira específica, na gravidez denominada de alto risco.

Por serem considerados a maior expressão do risco gravídico para o concepto, estes conceitos de prematuridade e de insuficiência ponderal vão exercer importância fundamental na área Materno-Infantil articulando à Obstetrícia a Pediatria, através de um novo campo que se delineia na década dos anos 50 denominado de Perinatologia. Essa relevancia pode ser atestada não só na literatura especifica sobre o assunto mas, principalmente, pela grande preocupação que cerca tanto organizações médicas (como, por exemplo, a Academia Americana de Pediatria), como também a Organização Mundial da Saúde que, nas décadas de 50 e 60, preocuparam-se com a questão da definição precisa destes termos com vistas à utilização dos mesmos como indicadores, ou fatores, de risco para as mortalidades e morbidades neo-natal e infantil (ARENO, 1984; SIQUEIRA, 1981). Como falo relacionado obser- va-se que os mesmos passam, a part de então, a ser amplamente utilizados na área Maternc Infantil, dentro de uma perspectiva de Saúde Pública, pré curando articular, ao estudo de suas causas, as condiçõe sociais e económicas e aos seus efeitos, a questão d morbidade quer para o recémnascido, quer para a crianç no seu processo de crescimento e de desenvolviment (SIQUEIRA, 1981; ARENO, 1984; ALVARENGA 1984).

Em função dos próprios critérios utilizados para a suas definições estes dois conceitos apresentam-se, n literatura especializada, como o primeiro nível de prec cupação com relação à mensuração do risco na are Materno-Infantil; tratam-se de propostas de busca de dá terminação do ritmo de crescimento intra-uterino ei relação à idade gestacional. Na medida em que a deter minação deste ritmo representava uma possibilidade d predição da ocorrência quer da prematuridade, quer d insuficiência ponderal, o que se observa são as tentativa de criação de curvas ou tabelas de crescimento do cor cepto capazes de revelar um padrão de cresciment intra-uterino normal, que funcionaria, não só como ind cador de risco de prematuridade e de insuficiênci ponderal mas também, por extensão, como risco neo-n tal e infantil atrelado às questões de crescimento e d desenvolvimento da criança^2, este último estreitament voltado para a noção de danos neurológicos. Como exen plos clássicos destacam-se, neste particular, trabalhe como os de LUBCHENCO et al (1963); CLIFFORI (1964); SPERS \& WACHOLDER (1982); STARFIELI et al (1982) e SCHWARCZ et ai (1987), dentre outros.

Assim colocado, este tipo de proposta de mensura ção do ritmo de crescimento intra-uterino revela-se d grande importância, não só por traduzir o esforço present nas clínicas Obstétricas e Pediátricas, ou mais especifi camente na Perinatologia - no sentido de elabora instrumentais que dessem conta dos problemas da áre dentro de uma perspectiva de Saúde Pública - mas tam bém por representar uma precursora das própria propostas de mensuração do risco gravídico3. Estas últi mas, caracterizadas por "sistemas de escore” ou d “pontuação”, são elaboradas notadamente pela clínic Obstétrica visando a determinar não só qs níveis-de risco de morbidade e de mortalidade materna mas, sobretudc 0 risco perinatal representado particularmente pelas pra babilidades de ocorrência de prematuridade e de insufi ciência ponderal do concepto, também entendida esta última como retardo de crescimento intra-uterino.

2 Sobre a questão da utilização de curvas ou tabelas visando a determinar o risco de peso inadequado ao nascer veja-se, por exemplo, SIQUEIRA (1981).

3 A propósito de uma análise teórico-metodológica e de aplicação das propostas de mensuração do risco gravídico na área maternoinfantil veja-se Tese de Doutorado de ALVARENGA (1984). 


\section{CONTEXTO DE EMERGÊNCIA DAS PROPOSTAS DE MENSURAÇÃO DOS NÍVEIS DE RISCO GRAVÍDICO: MOVIMENTO DA MEDICINA COMUNITÁRIA DOS ANOS $60 \mathrm{E}$ SUA RELAÇÃO COM A SAÚDE PÚBLICA}

Todas estas propostas de mensuração terão grande aplicação no campo da Saúde Matern Infantil a partir da decada de 60, principalmente em funçao da perspectiva que se abre para a incorporação da prática médica no interior do campo da Saúde Pública4. Por outro lado, a prática médica, através do seu movimento de recomposição chamado de "Medicina Comunitária”, aproxima-se da Saúde Pública em termos do interesse no mesmo objeto, ou seja, nos grupos vulneráveis a agravos de saúde ou, mais especificarnente, nos grupos de risco (ALVARENGA, 1984).

Isto pode ser especialmente evidenciado quando se considera, com DONNANGELO (1976), que a caracteristica básica que norteia a proposta de "Medicina Comunitária” é a de procurar localizar os elementos responsáveis pela inadequação da prática médica para a solução dos problemas de saúde não apenas nos aspectos internos do ato médico individual, mas especialmente em aspectos organizacionais da estrutura da atenção médica. Isto visando a superar essa inadequação através de novos modelos de organização, que tomassem como base o cuidado dos grupos populacionais, antes que a dos indivíduos. Neste sentido e que tal autora afirma que "é como prática de saúde, como forma de prestação de serviços, antes que como um campo sistematizado de princípios que a Medicina Comunitária se origina nos Estados Unidos - na década de 60- e se difunde na década de 70 - para as sociedades dependentes com o suporte das agencias internacionais, tais como a Organização Mundial de Saúde e a Organização Panamericana de Saúde” (ib. p. 85).

Aspecto importante é o de que a esta virá agregar-se os princípios básicos que norteiam dois outros movimentos de recomposição da pratica médica anteriores, ou seja, o da "Medicina Preventiva" e o da 'medicina Integral”, ocorridos na primeira metade do século ${ }^{3}$. Como propósito básico, a "Medicina Comunitária” destina-se a prestação de serviços às categorias excluídas do cui- dado médico. Enquanto tal, constitui-se numa forma alternativa releia à organização dominante da assistência e potencialmente capaz de compatibilizar o aumento de consumo de serviços e a questão dos altos custos da assistência médica através de propostas de recomposição dos próprios meios de trabalho (DONNANGELO, Estas propostas, caracterizadas especialmente por de simplificação das técnicas de atendimento má consideradas inovadoras e visando a uma amplia, consumo permitem a este projeto "Medicina Comunitária” configurar-se como uma nova modalidade de organização Interna da prática aonde a perspect existência de elementos racionalizadores da mesmas passe a ser fundamental. Isto porque tanto esta simplificação de técnicas, como a nova modalidade de organização interna da prática, procuram atender particularmente aos interesses do Estado, no que respeita à necessidade de fazer frente aos problemas de Saúde Pública, giz sobretudo pelas categorias sociais excluídas do c médico, cuja significarão "pública” passa a ser $r$ mente política. Neste particular destaca-se o Materno-Infantil pela alta vulnerabilidade que apresenta e por se constituir, a mortalidade e a morbidade perinatais num dos principais desafios a serem enfrentada setor saúde de então.

Inserida neste contexto, pode-se constatar (ALVARENGA, 1984) que as propostas de mensuração do risco gravídico ernergem no interior da prática médica tobstétrica buscando satisfazer a duas condições: a pririmeira a de colocar o conceito de risco entendido generic;amente como "a probabilidade de ocorrência de um dano” - tanto como uma "forma de conhecimento" da quase totidade dos fenômenos presentes na área, quanto uma estratégia de intervenção para a "solução" desses mesmos problemas. A segunda, inscrever esse conceito num conceito teóricometodológico capaz de dotá-lo de possibilidades ou mais especificamente, da propriedade em artucular num sistema logicamente coerente, os vários aspectos pertinentes à problemática básica presente no interior di campo da saúde, mas equacionada no nível da soctedade inclusiva, que se refere à questão da inadequação das práticas desse campo para atender às necessidades de saúde (pública ou coletiva) da população (ALVARENGA, 1984). Como decorrência, este conceito va poder transitar, em função do nível de abstração do sistema teórico em que se insere, tanto nas questões orgânicas

4 No trabalho de ALVARENGA (1984), principalmente no item 4.1., o leitor poderá observar o processo de ampliação c delimitação progressiva que sofre o campo da Saúde Pública em toda a primeira metade do século e que culmina, nos anos 60, com um movimento característico denominado de Integração Sanitarista”, que muito se aproxima dos objetivos a que se propõe, por outro lado, a própria medicina com seu movimento de recomposição da prática médica denominado "Medicina Comunitária”. Para uma análise especifica do movimento de “Integração Sanitária” veja-se Tese de Livre Docência de RAMOS (1972).

5 Para um tratamento especifico desses dois movimentos veja-se trabalhos de AROUCA (1975) e de DONNANGELO (1976). Para uma análise das relações desses movimentos com a emergência do conceito de risco na área Materno-Infantil, veja-se Tese de Doutorado de ALVARENGA (1984). 
relativas às saúdes da mulher e do concepto, quanto nas de natureza estruturais, tais como, os altos custos da assistência médica e a necessidade de racionalizar os recursos existentes; este fato acarreta sérias questões para o processo de conhecimento porque lhe imprime, do ponto de vista metodológico, problemas de vagüidade por não se conseguir, no campo científico, atribuir um limite à classe dos objetos ou coisas que o conceito denota. A isto equivale dizer que, com o mesmo referencial teórico-metodológico, tal conceito é aplicado quer às questões clínicas, específicas da área médica, quanto às questões econômico-financeiras, próprias das áreas das ciências sociais presentes no campo da Saúde Pública ou Coletiva.

No entanto, as implicações deste tipo de proposta de abordagem do risco gravídico vão mais além. Isto porque vai permitir, ao possibilitar, do ponto de vista técnico, rastrear mulheres de alto risco, conciliar e justificar os interesses de uma medicina tecnológica - de alto custo - com os de uma medicina simplificada, inscrita nos preceitos da Medicina Comunitária, e mais articulada com os interesses e possibilidades de atuação do tradicional campo da Saúde Pública, aonde a questão da racionalização dos recursos, ao lado de uma ampliação da cobertura sobretudo à população "carente" colocam-se como aspectos tomados como essenciais (ALVARENGA, 1984).

Observa-se aí um dos problemas básicos do uso da técnica, enquanto ideologia, na medida em que esse conceito, no esquema teóricometodológico em que opera, permite atender a interesses de diferentes ordens, tais como, os econômicos e ideológicos, sobretudo, em função da maneira como é tipicamente organizado, em lermos operacionais, com base em dada proposta de "sistemas de escore”, "sistemas de notação” ou, ainda, "tabelas de risco".

Apresentada de uma maneira característica, essa tendência em trabalhar com sistemas de escore vai responder a uma preocupação dos profissionais da Obstetrícia em mensurar o risco gravídico visando com isso não só ampliar a sua aplicação, mas também atribuir-lhe um estatuto científico dentro de uma concepção de ciência “positivista”.

No interior desses sistemas de mensuração o risco gravídico é concebido, pois, como "a probabilidade de ocorrências de um dano” representando, portanto, os termos 'probabilidade' e 'dano' suas características essenciais.

Na medida em que para operar no processo de investigação tais conceitos necessitam inscrever-se num dado corpo teórico (e metodológico) é que se pode observar suas articulações a outros como "fator de risco" (concebi- do como simples “indicador” ou "causa” de risco mas empregado, frequentemente, de maneira indistinta) e "graus" ou "níveis de risco", que facultam ao investigador diferenciar e caracterizar o conceito de "alto risco gravídico”, em relação aos de "médio" e "baixo" níveis.

Embora logicamente coerente este esquema teórico-metodológico apresentará, em seu emprego empírico, implicações de diferentes ordens, o que permite identificar uma série de problemas epistemológicos de grande relevância para tratamento do risco, enquanto objeto de preocupação do Campo da Saúde em geral e da Área Materno-Infantil em particular.

\section{CARACTERÍSTICAS BÁSICAS DOS SISTEMAS DE ESCORE E IMPLICAÇÕES TEORICO-METODOLÓGICAS NOS NÍVEIS DO CONHECIMENTO E DA PRATICA NA ÁREAMATERNO-INFANTIL}

No levantamento bibliográfico para a tese de doutorado, que compreendeu o período de 1959-1982 de literatura especializada, ALVARENGA (1984) observa que, para o processo de construção desses sistemas de escore, os autores partem de uma concepção de “causalidade múltipla”, o que lhes permite, raciocinando com PLAUT (1984), uma saída da "multicausalidade". Com esta, os vários fatores selecionados para compor o instrumento de mensuração interrelacionamse mas podem apresentar probabilidades desiguais em função da atribuição de pesos ou notas aos mesmos. Como resultado, é pressuposto que esse conjunto de fatores somam-se para a produção de determinado efeito ou resultado indesejado. Desse modo, o escore obtido com a aplicação do sistema elaborado passa a representar a "causa total" que responde por determinado dano destacando-se, entre eles, os da prematuridade, da insuficiência ponderal e da morbimortalidade perinatal. Quanto à concepção de risco presente esta refere-se a do "risco relativo".

Considerando que em todo processo de mensuração regras metodológicas (tanto teóricas, como técnicas) estão presentes para se poder definir o que se quer medir e, sobretudo, aquilo que se pode medir, o que se constata é que a não observância dessas regras, ou convenções, no caso da construção dos sistemas de escore para a mensuração dos níveis de risco gravídico, passa a gerar sérios problemas para a “avaliação científica” desses instrumentos o que repercute, quer nas possibilidades de conhecimento gerado, quer na utilidade prática dos mesmos em termos de intervenção no campo da Saúde Materno-Infantil. 
Ao construírem em bases totalmente arbitrárias e isentas de validade a maioria dos sistemas de escore, o que fazem os autores nesse campo da produção chamada científica é ignorar a grande tradição presente nas ciências humanas, representada pelas áreas das escalas sociométricas e também psicométricas, que vêm se ocupando da construção de instrumentos de medidas similares, tais como, escalas, índices, dentre outros.

A consideração de alguns aspectos do processo de construção desses sistemas de mensuração permite reter as principais questões metodológicas neles presentes e que desafiam cientistas com esse nível de preocupação.

A primeira delas refere-se ao processo de seleção das variáveis ou fatores de risco que comporão o sistema de escore.

O que se observa, nesse caso, é a ausência de consenso entre os pesquisadores, quer em relação aos fatores de risco que devem compor o instrumento de medida em função de sua relevancia como indicador ou causa, quer em relação ao número destes a serem selecionados. Como decorrência, o que se apresenta na literatura especializada são sistemas de escore ora extremamente complexos, ora extremamente simplificados.

Pato a destacar em relação a este aspecto é o de que, embora as várias propostas de mensuração explicitem preocupação por danos ou agravos específicos, tais como, a prematuridade, a insuficiência ponderal (ou retardo de crescimento intra-uterino) e a morbimortalidade perinatal acabam, na realidade, por apresentarem um conjunto similar de fatores de risco o que, ao lado de demonstrar um não recurso a um quadro de referência teórico que norteie tal seleção, parece também indicar os próprios limites de conhecimento científico ainda existentes no campo da Perinatologia, cuja complexidade manifesta-se na busca de sua interface com a Obstetrícia.

Revelando ainda essa arbitrariedade e desconhecimento metodológico (teórico e técnico) em relação à seleção de variáveis coloca-se, igualmente a tendência de muitas tabelas de risco conterem, em seu interior, um conjunto de variáveis de diferentes naturezas que representam várias dimensões do fenómeno ou dano a ser medido. Assim, nestes sistemas de escore são freqüentemente consideradas como pertencentes ao mesmo conjunto de fatores de risco tanto as variáveis de natureza orgânica, quanto as de natureza social e psicológica.
Como decorrência o que se observa é a existência de processos de redução tanto do social, quanto do psicológico a uma conexão causal que se supõe existir para as variáveis de natureza biológica descaracterizando totalmente as especificidades próprias que cada uma dessas dimensões possui em relação ao problema, ou dano, investigado.

Este procedimento gera, em relação ao social, serias implicações de natureza teóricometodológica uma vez que toda a historicidade do mesmo é ignorada e este passa a apresentarse, no interior do sistema de escore, fragmentado em variáveis, escamoteando, com isso, sua verdadeira hierarquia de determinação do processo saúdedoença e os desdobramentos analíticos que tal processo encerra, em termos de uma abordagem interdisciplinar ${ }^{6}$.

Outra questão metodológica importante em relação à construção dos sistemas de escore refere-se à tendência arbitrária igualmente observada com relação à atribuição de pesos (ou notas) às variáveis de risco que passam a representar os diferentes graus de “determinação” do dano.

Calcado no julgamento clínico e individual do pesquisador os graus de probabilidade dos fatores de risco em relação ao evento sob análise são determinados dentro de uma concepção subjetiva simplificada, basicamente desprovida de critérios metodológicos, quer teóricos, quer técnicos, que Ihes dêem fundamento.

Em relação à essa questão da atribuição subjetiva de pesos às variáveis de risco vale destacar que a literatura sobre a mensuração de risco tecnológico tem avançado na discussão. ALVARENGA (1984) identificou, por exemplo, na Rèvista Risk Analysls, editada a partir de 1980, a existência de uma grande polémica travada entre os defensores de uma postura objetivista de probabilidade (baseada na lei dos grandes números) e aqueles que trabalhando com eventos raros (caso, por exemplo, das usinas nucleares), passam a defender e a fundamentar não só a existência, mas também a necessidade de ampliação do conceito de probabilidade, atualmente muito circunscrito à teoria “objetivista” ou "freqüencialista”; tomam como esquema de referência uma postura subjetivista representada por uma teoria “compreensiva” da probabilidade, cuja concepção teórica prende-se à tradição bayesiana ${ }^{7}$.

6 A identificação do tratamento do processo saúde-doença perinatal reduzido a uma eoncepcão biológicA, abstraTa, pontual e fragmentada de “dano” encontra lugar e expressão na crítica teórico-metodológica que LAURELL (1983) e BREILH \& GRANDA (1980) fazem à tradicional abordagem epidemiológica dos “eventos de saúde”, por terem uma concepção histórica e dialética do social a partir da qual o conceito de "determinação” do dano redefine-se em termos da existência do processo saúde-doença com diferentes hierarquias de "determinação".

7 Para um tratamento introdutório à teoria subjetiva de probabilidade de Bayes veja-se por exemplo, ROBERTS (1974). 
O que se depreende dessa polêrnica é que não é a adoção. de uma postura subjetiva na elaboração dos sistemas de mensuração do risco gravidico que se constitui num problema epistemológica, mas sim a ausência de qualquer tipo de preocupação com regras metodológicas já existentes para esse tipo de postura compreensiva, fundamentadas e defendidas por autores dessa corrente como BLACK (1972) e APOSTOLAKIS (1978), dentre outros ${ }^{8}$.

Uma última questão metodológica relevante, que vale destacar em relação à construção dos sistemas de escore, encontra-se estreitamento relacionada ao problema da determinação dos graus de probabilidade, que se apresentam numa escala de 0 a 1 , sob a forma de um continuam. Trata-se da determinação dos estratos que definem como o instrumento de medida vai classificar os níveis de risco em baixo, médio e alto.

Freqüentemente definidos de maneira arbitrária os "pontos de corte" nos sistemas de escore apresentam-se como questão fundamental na medida em que são os níveis ou amplitude de estratos que eles definem, que dão a medida da relação existente entre o diagnóstico, o prognóstico e o resultado obtido. Por outro lado, é a partir desses níveis que se estabelece o grau de "validade" do instrumento, ou seja, a sua "fidelidade diagnóstica".

A respeito da questão o trabalho de FORTNEY \& WHITEHORNE (1982) é bastante esclarecedor. Demonstra que um "índice (ou tabela) de alto risco não é válido se uma proporção de pacientes de alto risco não é diagnosticada como tal ("falso negativo") ou se uma proporção de pacientes é definida como sendo de alto risco quando na realidade não o é( “falso positivo"). Daí enfatizarem que na avaliação da utilidade de qualquer instrumento de medida de risco as conseqüências do erro ("taxa”) de diagnóstico devem ser consideradas.

Tais conceitos são de grande significancia para o teste de fidelidade diagnóstica que considera igualmente dois outros elementos que desafiam o emprego de sistemas de escore nesse campo do conhecimento. Tratam-se dos graus de "sensibilidade" e de "especificidade" que tal modalidade de instrumento possui para a identificação da proporção de casos "falso negativos" e "falso positivos". Na literatura especializada a variação observada por autores como FORTNEY \& WHITEHORNE (1982) e LESINSKI (1975) para os primeiros foi de 0,7 a 11,3\% enquanto que, para os "falsos positivos", a mesma oscilou de 17,0 a 96,2\%. A diferenciação existente em relação à delimitação dos estratos de risco explicam também a variação constatada de $11 \%$ a $55 \%$ para o caso da proporção da população gestante diagnosticada como de alto risco pelas diversas propostas de mensuração.

\section{DESAFIOS METODOLÓGICOS PRESENTES PARAA PERINATOLOGIA COMO ÁREA DE INTERFACE NO CAMPO DA SAUDE MATERNO-INFANTIL}

Diante dessa ordem de problemas epistemológicos decorrentes do emprego do conceito de risco baseado nas propostas de mensuração por sistemas de escore, a área da Perinatologia passa a enfrentar desafios metodológicos - teóricos e técnicos - com implicações diretas para a relação teoria e prática.

Isso porque, dentro da "lógica” da proposta, 0 classificar erroneamente pacientes como de baixo risco quando na realidade o são de alto risco (falsos negativos` gera, no caso de pacientes necessitando de assistência especializada não a receber, o aumento da mortalidade ou morbidade materna, fetal e perinatal. Por outro lado ao classificar uma grande proporção de pacientes come sendo de alto risco, o instrumento passa a não atender às finalidades da proposta de abordagem de risco que, nó nível da administração de serviços, visa a transformá-le num instrumento de intervenção na busca de racionaliza os recursos existentes - sobretudo os escassos - e dis pensar assistência diferenciado em função daí modalidades ou graus de risco apresentados pelas pacientes (WHO, 1978; BACKETT et al, 1984).

Relacionado a esses aspectos colocam-se igualmente os problemas concernentes à forma "pragmatista" e "empiricista" de como as questões metodológicas - teóricas e técnicas - dos processos de mensuração são desconsideradas, por investigadores dessa área de conhecimento, em termos do paradigma de investigação que propõem.

Tendo em vista que os conceitos de prematuridade e de insuficiência ponderal - centrais na área da Perinatologia - representam, por um lado, a relação que $\sim$ mesma estabelece com a Obstetrícia - ao centrar nó organismo materno diferentes concepções de ritmos de crescimento intrauterino (do feto) e, por outro, a mediação que tal área opera entre a Obstetrícia e a Pediatria porque nesses dois conceitos centram-se os estudos daí causas sobre a questão do crescimento e desenvolvimento infantil (e pós-infantil) - é que se pode não somente observar a amplitude que tal campo de estudos abarca mas igualmente os desafios metodológicos nele presentes. Isso porque, se os limites da questão interdisciplinar já se colocam no nível do conhecimento biológico dessas diferentes áreas envolvidas nos estudos até agora produzidos, tal desafio amplia quando se considera necessidade de se avançar, para além do biológico, a \begin{tabular}{l}
\hline O leitor poderá encontrar a explicita^Sao de tal polémica em nível da introdução do trabalho de Tese de Doutoradn de ALVARENGA \\
(19841.
\end{tabular} 
análise das relações do nsco gravídico e pennatal para além da dimensão biológica, aonde a questão da interdisciplinanedade, a despeito de sua maior complexidade, perrnite apreender tais objetos em suas naturezas quer biológica, quer psicológica e também social.

Neste sentido, se a atual proposta de abor-

\section{BIBLIOGRAFIA}

ALVARENGA, A. T. de. O conceito de risco na área materno-infantil: considerações teóricas, metodológicas e de aplicação. São Paulo, 1984. [Tese de Doutorado - Faculdade de Saúde Pública da USP].

APOSTOLAKIS, G. Accident analysis: probability and risk assessment: the subjetivistic view point and some suggestions. Nud. Saf., 19(3): 305-15, 1978.

ARENO, P B. Contribuição ao estudo da antropometria do recémnascido. São Paulo, 1984. [Dissertação de Mestrado - Faculdade de Saúde Pública da USP].

AROUCA, A. S. da S. O dilema preventivista. Contribuição para a compreensão e critica da medicina preventiva. Campinas, 1975. [Tese de Doutoramento - Faculdade de Ciências Médicas da Universidade Estadual de Campinas].

BACKETT, E. M. et ai. The risk approach in health care: with special reference to maternal and child health including family planning. Geneva. World Health Organization, 1984.

BLACK, M. Probability. In: EDWARDS, R et ai. The enciclopaedia of philosophy. New York, Macmillan, 1972, v. 5, p. 464-79.

BREILH, J.; GRANDA, E. Investigacién de la salud en la sociedad: guia pedagogico sobre un nuevo enfoque dei método epidemio16gico. Quito, Ed. CEAS, 1980.

CLIFFORD, D.S.H. High-risk pregnancy. I Prevention of prematurity the sine qua non for reduction in mental retardation and other neurologic disords. New EngL J. Med., 27(5): 243-49, 1964.

DONNANGELO, M. C. F.; PEREIRA, L. Saúde e Sociedade. São Paulo, Ed. Duas Cidades, 1976.

FORTNEY, J. A.; WHITEHORNE, E. W. The development of an index of high-risk pregnancy. Amer. J. Obstet. Gynec., 143(5): 501-8, 1982. dagem de nsco apresenta limites para o processo de conhecimento e de intervenção na área Matemo-Infantil, tem o ménto de apontar para as diferentes ordens de desaf os que a esta se colocam e para a necessidade e a relevancia da comunidade científica investir intelectualmente no sentido de enfrentá-los.

LAURELL, A. C. A saúde-doença como processo social. In: NUNES, E.D. (org.) Medicina Social: aspectos históricos e teóricos. São Paulo, Ed. Global, 1983, p. 133-58.

LESINSKI, J. S. High-risk pregnancy. Unresolved problems of screening, management, and prognosis. Obstet. Gynec., 46(5): 599-603, 1975.

LUBCHENCO, L. O. et ai. Intrauterine growth as estimated from liveborn birth-weight data at 24 to 42 weeks of gestation. Pediatrics, 32: 793-800, 1963.

PLAUT, R. Analisis de riesgo, alcance y limitaciones para ei administrador de salud. BoL Of: Sanit. Panarner., 96: 296-304, 1984.

RAMOS, R. A integração sanitária: doutrina e prática. São Paulo, 1972. [Tese de Livre Docência - Faculdade de Saúde Pública da USP].

ROBERTS, H. V. inferência bayesiana. In: Enciclopedia Internacional de las Ciências Sociales. Madrid, Aguilar, 1974, v.5, p.781 -5.

SCHWARCZ, R. et ai. Principales causas perinatales de la mortalidad feto-neonatales y de las secuelas invalidantes en ei ni no, em pai ses latinoamericanos: algunas estrategias y acciones para contribuir a su abatimiento. In: FESCHINA, R.H. Tecnologias perinatales: fundarnientos, desarollo y evaluación. Montevidéo, CLAP/OPAS, 1987, p. 8-21 (Publicación Cientitica CLAP, 1122), 1987.

SIQUEIRA, A. A. E de. Indicadores de risco de peso inadequado ao nascer. São Paulo, 1981. ^CIese de Livre-Docência - Faculdade de Saúde Pública da USP].

SPIERS, RS.; WACHOLDER, S. Association between rates of premature delivery and intrauterine growth retardation. Dev. Med Child Neurol., 24: 808-16, 1982.

STARFIELD, B. et ai. Mortality and morbidity in infants with intrauterine growth retardation. J. Pediat., 10I: 978-83, 1982.

WORLD HEALTH ORGANIZATION Risk approach for rnaternal and child health care. Geneva, 1978. (WHO-Offset Publication, 39) 